undulatum Ehrh. Steierm., Voitsberg. - 1132. Eucladium verticillatum L. sub Bryo; Br. et Schimp. Tirol, Weiherburg bei Innsbruck. - 1133. Jungermannia Taylori Hook. Tirol, Lapones im Gschnitzthale. - 1134. Jungermannia riparia Tayl. Tirol, Padaster b. Trins. - 1135. Jungermannia minuta Crtz. Tirol, Padaster b. Trins. - 1136. Jungermannia albicans L. Tirol, Volderthal. - 1137. Bazzania triangularis Schleich. sub Jungermannia; Lindb. $=$ Jungermannia deflexa Mart. = Herpetium deflexum Nees a. $\mathrm{E} .=$ Mastigobryum deflexum Gottsch. Lindb. at Nees. = Pleuroschisma deflexum Dum. Steierm., St. Nikolai; Tirol, Trins im Gschnitzthale. - 1138. Grimaldia triandria Scop. sub Marchantia = Duvalia rupestris Nees. a. E. = Grimaldia rupestris Lindb. Krain, Zwischenwassern u. Idria. Mit einer Notiz von Lindberg. - 1139. Grimaldia fragrans Balb. Tirol, Guntschna b. Bozen. - 1140. Sauteria alpina Nees ab Es. sub Lunularia. Nieder-Oesterr., Klamm b. St. Egid a. Neuwald.

(Schluss folgt.)

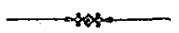

\title{
Iiteraturberichte.
}

Fura von Hernstein in Nieder-0esterreich und der weiteren Umgebung. Bearbeitet von Dr. Günther Beck. Wien 1884. Mit 11 Tafeln (von denen 3 colorirt sind), einer Cultur- und einer Forstkarte (letztere beide colorirt).

Dieses 288 Seiten Text in Gross 8. umfassende Werk ist ein Theil der mit Unterstützung Sr. kais. Hoheit des durchlauchtigsten Herrn Erzherzogs Leopold von M. A. Becker herausgegebenen Monographie "Hernstein in Nieder-Oesterreich, Wien, 1884“. In einer kurzen Einleitung gibt zunächst der Autor Aufschluss über die Veranlassung des Werkes zugleich bemerkend, dass ihm mit Rücksicht auf die bisher noch mangelhaften Arbeiten in Betreff der Kryptogamenflora Nieder-Oesterreichs nur ein verhältnissmässig kurzer Zeitraum (8 Wochen) zur Durchforschung des ausgedehnten Gebietes von Hernstein zur Verfügung stand, eines Gebietes, das sich über eine Fläche von ungefähr $1360 \square$ Kilometer oder ca. $24 \square$ Meilen erstreckt und ausser den Waldlandschaften am Piesting- und Triestingbach auch noch die Alpen- und Voralpenhöhen des Schneeberges und das flache Culturland zu beiden Seiten der Eisenbahn von Neunkirchen bis über Baden hinaus umfasst. Ungefähr in der Mitte des Florengebietes steht, von Schwarzwald umgeben, die Herrsehaft Hernstein.

Um aus der Flora dieses vielgliedrigen Gebietes ein wohlgeordnetes Ganzes zu schaffen, hätte es, unter anderen Umständen, noch weiterer mehrjähriger Forschungen bedurft. "Wenn ich mich gleichwohl", schreibt der Autor, "über all tiese Bedenken hinaussetzte, 
so war es vorwiegend die beruhigende Erwägung, dass ich bereits in den früheren Jahren bei zahlreichen botanischen Excursionen nach dem Schneeberg und dessen Umkreis auch einzelne Theile der Gegend von Hernstein in den Bereich meiner eingehenden wissenschaftlichen Forschungen gezogen hatte, und dass ich mich sonach in die angenehme Lage versetzt sah, die damals gewonnenen und in botanischer Hinsicht sehr werthvollen Wahrnehmungen, Aufzeichnungen und Messungen bei der gegenwärtigen Arbeit bestens zu benützen."

Der allgemeine Theil zerfällt zunächst in die zwei Abschnitte: I. Begränzung des Gebietes, II. die Vegetation im Allgemeinen. Letzterer gliedert sich folgendermassen: 1. Aufbau der Vegetation. 2. Landschaftlicher und botanischer Charakter, Verbreitung und Gliederung der im Gebiete vorkommenden Vegetationsformationen. I. Formation der Schwarzföhre; II. Formation der Buche; III. Formation der Fichte; IV. Formation des Voralpenwaldes; V. Formation der Erlen und Weiden; VI. Formation der Vorhölzer; VII. Formation der Legföhre; VIII. Formation der Wiesen; IX. Formation der Voralpenkräuter; X. Formation der Alpenmatten; XI. Formation der Felsenpflanzen. 3. Uebersicht der Bestandtheile der natürlichen Vegetationsformationen, worin der Autor die jeder einzelnen Formation eigenthümlichen Charakterpflanzen anführt und diesen auch jene Arten beifügt, welche zwar nicht ausschliesslich, jedenfalls aber mehr oder minder häufig in der betreffenden Formation angetroffen werden, wo es sich um Waldvegetation handelt, stets zwischen Oberholz, Unterholz und Niederwuchs unterscheidend. 4. Das Culturland. Künstliche Vegetationsformationen. Uebersicht der im Grossen gebauten Gewächse. Flora der Gärten. Es folgt dann ein Hauptabschnitt: III. Die Vegetation in pflanzengeographischer Hinsicht. A. Pflanzenregionen. 1. Die Region der Ebene und des Hügellandes; 2. Voralpenregion; 4., 5. die Krummholz- und Alpenregion. B. Vegetationsgebiete. Der Inhalt des IV. Hauptabschnittes ist ein geschichtlicher Rückblick auf die botanische Erforschung des Gebietes von Hernstein und Umgebung. - Der besondere Theil wird durch die Anführung der vom Autor benützten Quellenliteratur eingeleitet, dann folgt die Aufzählung der im Gebiete von Hernstein und dessen Umgebung beobachteten Pflanzen mit genauer Angabe ihres Vorkommens.

Wiewohl sich die "Flora von Hernstein" in der Art der Darstellung d. i. in der Gliederung des überreichen Stoffes, an Kerner's "Pflanzenleben der Donauländer" anschliesst, so sind doch die Schilderungen als naturgetreue, von einem lebhaften Natursinn getragene Wiedergabe des Gesehenen originell zu nennen. Dem Werke gereicht es sicher zum Vortheil, dass nur dort ein wärmerer Ton angeschlagen wird, wo das zu schildernde Naturbild es erheischt. Bekanntlich erscheint eine bilderreiche Sprache als schwülstig und überladen, wenn ihr bei Erörterungen von Fragen, zu denen nicht mehr als ein nüchternes fachmännisches Verständniss gehört, Raum gegeben wird. "Ein möglichst klares Bild der Vegetation von Hernstein und dessen Umgebung wollte ich hier schaffen," sagt der Au- 
tor, „ein im wissenschaftlichen Rahmen gehaltenes Naturbild, das die überraschende Fülle, Mannigfaltigkeit und Anmuth der Flora dieser Gegend getreu wiedergeben sollt $\theta_{.}$“ Und dieses ist demselben auch vollkommen gelungen. Jeder Freund der heimischen Flora wird ihm dafür Dank wissen. Aber auch derjenige, der sich nicht mit dem einfachen Verständniss und der Bewunderung des herrlichen Naturbildes in dieser anerkennenswerthen Darstellung begnügt, sondern tiefer ausblickt nach den Ursachen der so mannigfachen pflanzengeographischen Erscheinungen und Anomalien, wird das Werk mit Freude begrüssen, als eine Fundgrube der werthvollsten Details. So möchte ich insbesondere auf die Abschnitte 3,4 und 5 (S. 60-72) hinweisen, wo zahlreiche Daten über die obere und untere Grenze des Baumwuchses und über die obere und untere Grenze des Krummholzes am Schneeberge beigebracht werden.

Es ist hier nicht nur beachtenswerth, sondern geradezu auffallend, dass z. B. am Waxriegel, der 1884 Meter hoch ist, die Legföhre an der Südseite nur ca. 46 Meter höher hinaufsteigt als an der Nordseite. An der Südseite geht die obere Grenze des Krummholzes nur ca. 5 Meter über das Mittel, an der Nordseite bleibt sie nur ca. 41 Meter unter dem Mittel, während dieselbe am Schneoberge (2075 Meter) an der Nordseite ca. 60 Meter höher steigt, als am Waxriegel. Spricht das nicht deutlich genug für die mächtige Wirkung der bodenklimatischen Einflüsse, denen gegenüber der Einfluss der Lage gegen die Sonne beinahe gering zu nennen ist?

Das jedoch nur beispielsweise. Der Leser wird in dem inhaltreichen Werke Stoff und Anregung zu den belebrendsten Vergleichungen genug finden. Einen nicht geringen Vorzug gegenüber den älteren floristischen Arbeiton erblicken wir an der "Flora von Hernstein " auch darin, dass die Species im Geiste der neueren Systematik aufgefasst und in Anwendung gebracht wird, wodurch die Angaben im Vergleich zu den älteren phytographischen Daten erheblich an Brauchbarkeit gewinnen. Denn sie sind geeignet, die Grundlage zu weiteren Studien über die Geschichte der Formentwicklung der Pflanzen zu bilden, während mit Angaben, welche sich auf Cumulativspecies beziehen, dem in dem angedeuteten Sinne thätigen Forscher schwerlich gedient sein könnte.

Auch macht uns der Autor mit mehreren nenen Arten der Flora von Hernstein und Umgebung bekannt, die in seinem Werke beschrieben und sehr schön abgebildet sind: es sind Peziza atrofusca, $\boldsymbol{P}$. epichrysea, $\boldsymbol{P}$. imperialis, $\boldsymbol{P}$. limnophila, Dacrymyces multiseptatus, Ustilago Betonicae, Boletus Lorinseri, Euphrasia nivalis, Hieracium Neilreichii, $\boldsymbol{H}$. Breyninum, $\boldsymbol{H}$. orthophyllum, $\boldsymbol{H}$. digeneum nov. hybr., $\boldsymbol{H}$. interjectum nov. hybr. Ausser diesen sind auch noch abgebildet: Hieracium glaucoides M. F. Müllner, H. strictissimum Fröhlich, H. trichoneurum Prantl (glaucum $\times$ villosum), Thlaspi Goesingense E. v. Halácsy, Sorbus Hostii Jacq. fll., Rosa diversisepala H. Braun, Primula Portenschlagii Beck (P. Clusiana minima). 
Durch den schönen Druck, mehrere dem Texte ${ }^{1}$ ) beigefügte Holzschnitte (Kärtchen), die erwähnten sehr sorgfältig ausgeführten Pflanzenabbildungen, besonders aber durch die Beigabe von zwei grossen prachtvoll colorirten Karten, welche die Ausdehnung und Vertheilung des Waldes und des Culturbodens im Florengebiete veranschaulichen, erscheint das Werk geradezu luxuriös ausgestattet. So möge es denn, als eine Zierde der botanischen Literatur, in die Hände recht vieler Freunde der heimischen Flora gelangen.

Fr. Krašan.

Nägeli und Peter. Die Hieracien Mittel-Enropas. Monographische Bearbeitung der Piloselloiden mit besonderer Berücksichtigung der mitteleuropäischen Sippen. München 1885, 8. (Hfrz.) 931 pp.

Das Erscheinen dieses lange erwarteten Werkes wird allen Floristen hochwillkommen sein; einer Empfehlung beim botanischen Publicum bedarf ein solches classisches Werk wohl nicht, wohl aber verdient es, dass sich jeder Phytograph, selbst wenn er nicht beabsichtigt specielle Hieracien-Studien zu machen, möglichst eingehend mit ihm beschäftige. Das hier verarbeitete Material ist grossartig; im Laufe einer mehr als zwanzigjährigen Arbeit ist den Verfassern eine so grosse Anzahl unterscheidbarer und constanter Formen jedes Grades, von der schwächsten Varietät bis zur ganz abgesonderten Species, bekannt geworden, dass sie, um das Werk nicht ins Riesige zu steigern, eine Auswahl treffen mussten. Auch so noch ist die Zahl der unterschiedenen Arten auf 164 gestiegen, von denen die meisten eine grössere oder geringere Anzahl von Subspecies (bei H. Pilosella sind deren 108) Varietäten und Subvarietäten aufzuweisen haben, so dass die Zahl der hier beschriebenen Formen ("Sippen" wie die Verfasser sagen), gegen 2000 betragen mag.

Dieses riesige Material gesichtet, sorgfältig abgestuft und systematisch aufgebaut, endlich in klarer Weise beschrieben zu haben, so dass jedem, der ein ernstes Studium nicht scheut, ein sicherer Führer in das Reich dieser Formen an die Hand gegeben ist, dies ist das Hauptverdienst des gewaltigen Werkes, eines wahren Monumentes deutscher wissenschaftlicher Arbeit, dem niemand, der mit derartigen Arbeiten vertraut ist, seine Bewunderung versagen wird. Die Anordnung des systematischen Theiles ist folgende: das Subgenus Pilosella wird zunächst in die Acaulia und die Cauligera eingetheilt; die ersteren zerfallen in zwei, die letzteren in sieben Sectionen; innerhalb jeder derselben finden sich zunächst die Hauptarten (typischen Species) charakterisirt, dann erst folgen die Zwischenformeu und Bastarde innerhalb der betreffenden Section, dann jene mit den vorhergehenden Sectionen. Die Subspecies der polymorphen Arten werden der Uebersicht wegen zunächst zu „Greges" zusammenge-

1) Einige Versehen, die sich theils als Schreib-, theils als Druckfehler zu erkennen geben, werden der Verständlichkeit des Textes wohl kaum einen Abbruch thun, da sie der sachkundige Leser selbst berichtigen kann. 
fasst. Eine systematische Zusammenstellung sowie eine BestimmungsTabelle am Schlusse des Werkes werden die Uebersicht und das Arboiten erleichtern; ein auderer Abschnitt gibt die Bestimmungen der von verschiedenen Sammlern ausgegebenen Exsiccaten; die hervorragendste Sammlung ist die von Peter selbst ausgegebene, aus 300 Nummern bestehende.

Bisher haben wir uns nur mit dem systematischen Abschnitte beschäftigt, der insbesondere dem Floristen von Werth sein wird; die übrigen Abschnitte besitzen ein noch allgemeineres Interesse. Zunächst wird die Morphologie der Gruppe behandelt, ihre Innovations-Verhältnisse und einige biologische Charaktere besprochen; dann werden die einzelnen Merkmale in ihrem Verhalten in der Cultur untersucht, constante und veränderliche geschieden. In dem Abschnitte "die Species und ihre Bestandtheile" wird gezeigt, dass die "Sippen" ungleichen systematischen Werth baben, dass die Species theils aus Einzelsippen, theils aus Sippengruppen bestehen; es wird die phytogenetische Entstehungsweise der Sippen durch Variation und Divergenz, der Einfluss des Aussterbens von Sippen etc. an verschiedenen Beispielen und durch graphische Darstellung zu erläutern versucht. Bezüglich der systematischen Behandlung wird betont, dass nicht alle durch Zwischenglieder verbundenen Sippen zu einer Species zusammengefasst werden dürfen, weil dann die Endglieder solcher Reihen weniger untereinander verwandt wären als manche andere nicht durch Zwischenglieder verbundene Arten, der Mangel der letzteren hänge von einem zufälligen Umstande (Aussterben) ab. Die mannigfaltigen Beziehungen der Arten untereinander werden durch mehrere graphische Darstellungen versinnbildlicht. Ferner wird versucht festzustellen, wie sich die einzelnen Merkmale phylogenetisch entwickelt haben, welche von ihnen älterer, welche jüngerer Entstehung sind, und wird daraufhin auch oin Stammbaum der Piloselloiden angedeutet. Die Formenvermehrung durch Bastarde wird besonders behandelt und gezeigt, dass anf diesem Wege keine neuen Erscheinungen, sondern nur neue Combinationen schon vorhandener Merkmale erzengt werden und dass der Sippen-Bestand durch dio Bastarde wegen deren Seltenheit kaum geändert wird. Dio Bastarde werden übrigens im vorliegenden Werke wie "Zwischenspecies" behandelt und mit eigenen Namen belegt. Mit besonderem Interesse wird man das Capitel über geographische Verbreitung lesen, welches sich nicht auf die Piloselloiden beschränkt, sondern eine Skizze der Entstehung der mitteleuropäischen Flora überhaupt gibt. In besonderen $A b-$ schnitten wird noch über die Mittel zur Bearbeitung der Monographie, Literatur, Sammlungen, Reisen, Culturen, über Methoden der Bearbeitung und die Nomenclatur Rechenschaft gegeben. "Da die Ansichten der Systematiker über den systematischen Werth" der von den Verfassern unterschiedenen Sippen nach wie vor schwanken werden," so wurde mit Recht jeder Subspecies und besseren Varietät ein im Bereiche der Piloselloiden nicht wiederkehrender Name gegeben. 
Mit dem Speciesbegriff, wio ihn die Verfasser nehmen, werden allerdings nicht alle Systematiker einverstanden sein. Wäre die Ansicht der Verfasser, dass die Lücken zwischen den existirenden Arten nur durch Aussterben der Zwischenformen, also durch einen zufälligen Umstand entstanden sind, richtig, dann liesse sich allerdings gegen ihr Verfahren, aus einer Reihe gleitender Uebergänge zwischen zwei Arten Zwischenspecies gleichsam herauszuschneiden, die dann nach keiner Seite scharf begrenzt sind, nichts einwenden; denn wären die Uebergangsformen der jetzt isolirt stehenden Arten nicht ausgestorben, so müsste man dieselben eben mit in den Rahmen derselben aufnehmen; und würden z. B. innerhalb der Gattung Hieracium sämmtliche jemals entstandenen Formen noch existiren, so wäre das Unterscheiden von Species überhaupt nichts als ein willkürliches Zerschneiden des Formennetzes in annähernd gleichgrosse stücke. Zu dieser Consequenz führt wenigstens die Anschauung der Verfasser; denn dieselben sehen in jenen ganz schwachen Variationen, wie sie besonders bei Hieracien so vielfach vorkommen, Anfänge von Speciesbildung, und glauben, dass diese immer mit so kleinen Schritten begonnen habe, und die vorhandenen Lücken nur durch Aussterben zu erklären sind. Referent weiss sehr wohl, dass diese Anschauung, die ja auch Darwin hatte, bei der Mehrzahl der Forscher vorherrscht; dessenungeachtet möchte er daran erinnern, dass sie doch nur eine Vorstellungsweise ist, der sich die Thatsachen durchaus nicht immer nngezwungen fügen. Vielmehr deuten gar viele Erfahrungen darauf hin, dass die Speciesbildung nicht immer in so kleinen Schritten erfolgt sei, dass vielmehr allzeit und vielleicht besonders in gewissen Perioden auch raschere Umbildungen stattgefunden haben, so dass man selbst mit 0 . He er von einer "Umprägung der Arten" sprechen könnte. In diesem Lichte gewinnen dann jene Lücken zwischen den isolirten Arten eine höhere Bedeutung, und man darf solchen nicht ohne weiters Stücke von engverknüpften, also durch sehr kleine Schritte der Variation entstandenen Formenkreisen, als gleichwerthig setzen. Leider verbietet mir der Raum eines Referates, auf diese Dinge näher einzugehen, auch haben solche rein theoretische $\mathrm{Be}-$ denken nichts mit dem praktischen Werthe des vorliegenden Buches zu thun. In formeller Hinsicht möchte an demselben vielleicht befremdlich erscheinen, dass für die Species und die Greges lateinische Diagnosen, bald für sich allein, bald neben deutschen, für die Subspecies und Varietäten dagegen nur deutsche gegeben wurden; ein einheitliches Verfahren hätte dem Referenten besser geschienen. Die Ausstattung des Buches ist eine musterhafte.

E. Hackel.

Cohn Ferdinand, Beiträge zur Biologie dor Pflanzen. IV. Bd. 1. Hft. 4 Tafeln, Breslau 1884.

Das neneste Heft enthält folgende 3 Abhandlungen:

I. Ueber die Wasserbewegung in der Moospflanze und ihren Einfluss auf die Wasserströmung im Boden von Friedr. 0ltmanns. Desterr. botan. Zeitschrift. 5. Heft 1885. 
(Mit Taf. I-II.) Auf Grund ausgedehnter Untersuchungen über die Transpiration und Wasseraufnahme der Moospflanze und des Moosrasens im lebenden und todten Zustande gelangt der Verfasser zu dem Resultat, dass der Moosteppich des Waldes und der Moore wie ein Schwamm wirke, den man auf den Boden ausbreitet. Dor lebende Rasen wirkt ebenso wie der abgestorbene. Die Moose hemmen die Verdampfung des Wassers aus dem Boden, sie vermitteln ein langsames Eindringen des Regens in den Boden und halten auf abschüssigem Terrain das Wasser, welches bei heftigem Regen nur zu rasch abrinnen würde, mit grosser Gewalt zurück. Während die Mehrzahl der Moose das Feuchtbleiben des Bodens im hohen Grade begünstigt, verhalten sich die Torfmoose gorade umgekebrt, denn sie fördern, da sie die Oberfläche der verdunstenden Wasserfläche bedentend vergrössern, die Austrocknung der Moore.

II. Ueber Stephanosphaera pluvialis Cohn. Ein Beitrag zur Kenntniss der Volvocineen. Von Prof. G. Hieronymus. Mit Taf. III-IV. Durch diese Arbeit werden einzelne Lücken, welche die bekannten Arbeiten Cohn's und Wichura's über denselben Gegenstand offen liesen, ausgefüllt. Namentlich über die Bildung, die Beschaffenheit und die Copulation der sogenannten Mikrogonidien (Gameten) hat der Verfasser eingehende und erfolgreiche Untersuchungen angestellt.

IIl. Beitrag zur Entwicklungsgeschichte des pflanzlichen Zellkerns nach der Theiluug. Von Dr. Fr. Schwarz. Die Veränderungen, welche der Zellkern während der Theilung aufweist, sind von verschiedenen Beobachtern bis in die kleinsten Details untersucht worden. Um das weitere Schicksal des Kerns, um die Veränderungen, welche er nach der Theilung, also in der wachsenden Zelle erfährt, hat man sich bisher weniger bekümmert. Diess bewog den Verfasser zu dieser Untersuchung und er gelangte biebei zu folgenden wichtigeren Resultaten: 1. Die Tinctionsfähigkeit der Kerne variirt nach dem Alter. Junge Kerne färben sich stärker als alte. 2. In allen Geweben nimmt die Grösse der Zellkerne anfangs zu, dann später wieder ab. Analog verhält sich das Kernkörperchen. Hieraus und aus anderen Thatsachen schliesst der Verfasser, dass zwischen Kern und Zelle einerseits und zwischen Kernkörperchen und Kern andererseit ein Stoffaustausch stattfindet.

H. M.

Borbás Vince, Uj körisfa hazankban (Eine neue Fraxinusart in Ungarn). „Erdészeti Lapok" 1885, p. 165-167.

Fraxinus rostrata Guss. var. emarginata Strobl, welche Hirc im Dragathale entdeckte (0e. B. Z. 1884, p. 83) kommt auch im Vinodolthale bei Novi vor. Die stark zugespitzten kleineren Blätter scheinen an der Basis verhältnissmässig breiter und mehr abgerundet zu sein, als bei $F$. excelsior. Der Griffel der $F$. rostrata ist bei uns nur $2 \mathrm{Mm}$. lang. Ich glaube, sie ist eine südliche und Parallelform 
der $\boldsymbol{F}$. excelsior. Von $\boldsymbol{F}$. oxycarpa und $\boldsymbol{F}$. oxyphylla weicht sie durch die graubraune, nicht schwarze Knospe ab. Für den Karst ist die $F$. rostrata um so wichtiger, als sie ein kleiner Baum oder Strauch bleibt, da es nothwendig ist, dass hier eine Strauch- oder Macchionformation den Boden für den Wald vorbereite.

\section{v. Borbás.}

Bilder-Atlas des Pflanzenreiches von Professor Dr. Moritz Willkomm in Prag. Lieferung I und II à Mrk. 1:50. Verlag von J. F. Schreiber in Esslingen 1884. Gr. 4. Vollständig in 9 Lieferungen.

Wir begrüssen das Erscheinen des vorstebenden Werkes mit Freuden und dies umsomehr, als der bekannte Verfasser in klarer und streng wissenschaftlicher Weise mit Zugrundelegung des natürlichen Systemes uns durch Wort und Bild in die Pflanzenwelt einführt. In der ersten Lieferung finden wir nach einigen allgemeinen Bemerkungen über das Linné'sche System, das Schema eines natürlichen Systemes, welchem das von Endlicher als Basis dient, von dem Verfasser aber den Ergebnissen der neueren morphologischen Forschungen entsprechend umgeändert wurde. Hierauf folgt eine $\mathrm{Be}$ schreibung der wichtigsten Arten der Pilze, Flechten, Algen, Moose, Farne und Coniferen, dem sich in der zweiten Lieforung Vertreter der Ordnungen Lemnaceae, Typhaceae, Pandaneae, Palmeae, Gramineae, Cyperaceae, Alismaceae, Hydrocharideae, Butomeae, Musaceae, Orchideae, Iridea und Amaryllideae anschliessen. Die jeder Lieferung beigegebenen, in Farbendruck ansgeführten 8 Tafeln, onthalten dio fast durchwegs sehr gelungenen Abbildungen der oben citirten Arten, sowohl was Zeichnung als Colorit anbelangt. $\mathrm{Zu}$ wünschen bliebe nur, dass die Bezeichnungen auf don Tafeln mit den im Texte Bezughabenden einer eingehenden Correctur unterzogen würden, um den Laien vor leicht möglichen Irrthümern zu schützen; ebenso die Hinzufügung der Autoren-Namen bei den beschriebenen Arten. Der Verlagshandlung gebührt für die äusserst elegante Ausstattung des vorliegenden Bilder-Werkes volles Lob, umsomehr als es durch den staunend billigen Preis jedem sich hiefür Interessirenden leicht zugänglich gemacht wird.

J.

A. Ernst, "El Guachamaca" (Sep-Abdr. aus: "La Exposicion Nacional de Venezuela en $1883^{\circ .}$. Verlagsort: Caracas 1885).

Unter dem obigen - sowie unter dem Namen "Guaricamo" kennt man in Südamerika eine Drogue, die als Medicament und andererseits als sehr heftiges Gift in einem ungewöhnlichen Rufe steht.

Die Provenienz dieser Drogue war bis in die neueste Zeit eine zweifelhafte. Nach Humboldt wäre die fragliche Mutterpflanze die Ryana coccinea; nach Kunth eine Patrisia ( $P$. dentata?). Ramon Paez und nach ihm R. de Grosourdy beschrieben die vermeintliche Pflanze. - Letzterer (im Archiv der Pharmacie 1865) als eine Species der Prestonia. Erst den Forschungen Müller's gelang es, zu constatiren, dass man es mit einer Apocynee: Melonetia nitida Spruce zu thun habe. Der Verf. beschreibt die pharmakologischen 
Versuche, welche mit der genannten Drogue an verschiedenen Thieren unternommen wurden. Deren Ergebniss besteht darin, dass das betreffende Narcoticum ein dem Curare ziemlich analoges Verhalten zeigt, jedoch viel schwächer reagirt, und dass die Intensität der Wirkungen auf thierische Organismen sehr von der Jahreszeit abhängt, wo das Experiment gemacht wird.

Próíh oda.

Meehan Thomas, ,0pposite Leaves in Salia nigra (Marshall). Aus den Proceedings of the Academy of Natural Sciences of Philadelphia. P. II. 1884 (p. 151).

Die genannte Weide gehört zur Gruppe der Coëtaneae, deren Kätzchen nicht sitzend sind, sondern an der Spitze von kurzen, neugetriebenen Aestchen stehen. Die Kätzchen sind aber nicht absolut endständig, sondern erscheinen nur als solche wegen des zeitweiligen Zurückbleibens der Terminalknospe. Sobald nun die letztere nach dem Eintritte der Fruchtbildung an dem weiblichen Amentum hervorbricht und sich ein zweiter Trieb bildet, so wird das Fruchtkätzchen seitenständig, und in diesem Falle kann man beobachten, dass consequent das erste neue Blätterpaar gegenständig ist, während schon die nächsten ihre normale (abwechselnde) Anordnung besitzen.

Př́íhoda.

\section{Correspondenz.}

Lemberg, am 31. März 188 ö.

Es mögen noch folgende interessantere Daten aus der, Flora Galiziens dahier verzeichnet werden: Bromus erectus Huds. Sniatyn (exs. Hankiěwiez); Cerastium triviale var. vemorale Uechtr. Sinków (exs. Hank.); Cineraria longifolia DC. Buczacz (exs. Trusz.); Epilobium montanum $\times$ adnatum Cygany; Galium erectum anct. austriac., Anhöhe "Chomiec" bei Lemberg; Gatium aristatum mihi (non L.), Sinków (exs. Hank.); Heracleum Sphondylium L. Pieniny (exs. Stelzer); Iris sibirica L. Gródek (exs. Buschak); Lathyrus tuberosus Sinków (exs. Hank.); Luzula pallescens Bess. Romanów bei Bóbrka. (In Siebenbürgen kommt diese Art auch vor, aber Schur hat sie von $L$. multiflora nicht unterschieden. Plantago maior L. f. nana, Sinków (exs.) H a n k.); Poa hybrida Rchb., Lukawiec bei Bortniki (exs. Tiniecki); Pulsatilla grandis Sinków (exs. Hank.); Scorzonera purpurea Sinków (exs. Hank.); Silene densiftora Otth. Kołodróbka (exs. Hank.); Valeriana sambucifolia Mik., Pieniny (exs. Stelzer); Valeriana tripteris Pieniny (exs. Stelzer). Die von mir aus Lemberg angegebene Crepis foetida ist eigentlich $\mathrm{Cr}$. rhoeadifolia M. B.; auch Calamintha adscendens mihi stellt nicht die Pflanze Jordan's und Galium aristatum mihi nicht die Pflanze Linné's gleichen Namens vor, wie ich mich vor Kurzem überzeugt habe, nachdem ich Calamintha adscendens Jord. 